\title{
A Low-Interference Relay Selection for Decode-and-Forward Cooperative Network in Underlay Cognitive Radio
}

\author{
Chih-Wen(Wenson) Chang*, Po-Hsun Lin ${ }^{\sharp}$ and Szu-Lin Su ${ }^{\dagger}$ \\ $* \sharp \dagger$ Institute of Computer and Communication Engineering, \\ National Cheng Kung University, Taiwan
}

\begin{abstract}
In the underlay decode-and-forward (DaF) cooperative cognitive radio (CR) network, an optimal relay can be selected by the conventional max-min selection on the condition of not violating the interference temperature (IT) limit. However, the max-min selection may cause some extra amount of interference to the primary system (PS) such that the so-called transfer ratio (TR) can be lower. Note that TR is newly defined as the ratio of the secondary system's (SS's) capacity gain to the PS's capacity loss due to the activities of SS. In order to improve the TR value, we are motivated by the pricing function in Game theory to propose a novel low-interference relay selection by taking the impacts of the interference from SS to PS into consideration. Using the low-interference selection, however, the optimal relay may not always be picked. To clarify this phenomenon, the still optimal probability is defined as the probability of selecting the optimal relay by the proposed scheme. In addition, the impact of the low-interference selection on the SS's capacity is also analyzed. The simulation results prove not only the exactness of the analytical results but also the superior performance in terms of the TR value and total capacity which also indicates that a higher spectrum efficiency can be achieved. It is believed that the results of this paper can provide an alternative viewpoint of evaluating the spectrum efficiency and inspire more interesting and important research topics in the future.
\end{abstract}

Index Terms-Cognitive radio, decode-and-forward, cooperative network, transfer ratio, still optimal probability.

\section{INTRODUCTION}

Since the Federal Communications Commission (FCC) has revealed the truth of spectrum inefficiency in the incumbent wireless communication systems in 2002, cognitive radio (CR) has been regarded as one potential technology to activate the utilization of spectrum resources in the recent evolution of wireless communication systems [1]. Based on the definitions of spectrum holes in [1] and the operation modes in [2], [3], the overlay and underlay modes can be developed to use the white and gray spectrum holes, respectively. Literally, a white spectrum hole is a vacant spectrum segment, while a gray one is a spectrum segment partially occupied by the incumbent systems. Thus, in the underlay modes, an interference temperature (IT) limit should be imposed on the CR system such that the incumbent one can not be seriously affected. Accordingly, a CR system can arbitrarily boost its own capacity on the condition of not violating the IT limit [4].

To further enhance the system performance, a cooperative relay network can be incorporated into secondary system (SS). Thus, in the underlay CR system with an IT limit, the cooperative relay networks can also be applied to have a better capacity and error rate performance [5], tradeoff between achievable rate and network lifetime [6], maximum signalto-interference-plus-noise ratio (SINR) at the destination node [7], better channel utilization by multihop relay [8], maximum

\footnotetext{
${ }^{1}$ The contact author's e-mail: cwchang@ee.ncku.edu.tw

${ }^{2}$ This work was supported by the National Science Council, Taiwan, under the contract 97-2221-E-006-085-MY3.
}

throughput and reduced interference via beamforming [9], and maximum SINR using cooperative beamforming [10]. (A detailed literature survey is provided in [11]) From the literature survey, one can find that in the underlay cooperative CR network, the spirit of selecting an optimal relay based on either criterion can be popularly accepted as long as the IT limit can be satisfied. However, the very truth is in the underlay CR system, primary system (PS) should somehow sacrifice himself to tolerate a higher level of interference even though the activities of SS are restricted to satisfy the IT limit. For example, PS may need to increase transmission power to maintain the same quality of service (QoS), or have a degraded but acceptable QoS using the same transmission power. Thus, we are curious about one interesting question: how worthy of the PS's sacrifice in the underlay cooperative CR network? Can it be more valuable? If the answer is yes, how can we select a relay alternatively?

In this paper, we answer these questions by considering a decode-and-forward $(\mathrm{DaF})$ cooperative network in an underlay CR system. As shown in Fig. 1, on coexistence with a PS in the downlink infrastructure mode, there are numerous relays between the secondary user's (SU's) transmitter and receiver. Then, a relay can be a candidate one if the IT limit can not be violated. Now, the question is how to select a relay to rise the worth of the aforementioned PS's sacrifice. To evaluate this kind of sacrifice, a transfer ratio (TR) is newly defined as the ratio of the SS's capacity gain to the PS's capacity loss due to the activities of SS. Motivated by the pricing function in Game theory, which is usually defined as the difference between utility and cost functions to characterize the conflict of interests between users [12], an additional term reflecting the interference from SS to PS is taken into consideration as a cost function of relay selection. Also, the utility function is defined the same as that in the conventional max-min selection. Hence, in the SS's aspect, the optimal relay with the max-min link quality may not always be selected; and consequently, SS can only attain a lower capacity.

To clarify this phenomenon, the still optimal probability $\left(P_{S O}\right)$ is defined as the probability of successfully selecting the optimal relay by the proposed low-interference scheme. With the help of $P_{S O}$, the impacts of the low-interference selection on the SS's capacity can also be derived. In the overall aspect, however, the simulation results prove that the proposed low-interference relay selection can own a higher TR value and total capacity than those of the conventional max-min policy. This means that a higher spectrum efficiency can be achieved by the proposed method in terms of the TR value with no need of any extra costs, since the information requirement is the same as the conventional max-min policy (the issue regarding the required information of implementing the proposed scheme will be explained in Section III). It is believed that the results of this paper can provide an alternative viewpoint of evaluating the spectrum efficiency and inspire 
more interesting and important research topics in the future.

The rest of this paper is organized as follows. The system model is provided in Sections II. In Section III, we propose the low-interference relay selection scheme. Also, the associated $P_{S O}$ and its impacts on the SS's capacity are derived. The numerical results and concluding remarks are provided in Sections IV and V, respectively. Some discussions can also be found in Section V.

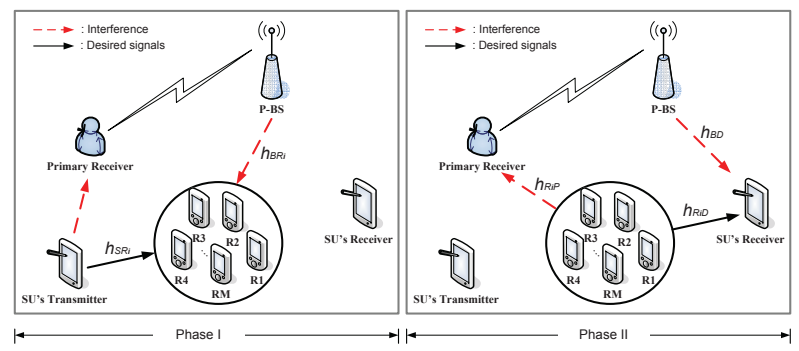

Fig. 1. A dual-hop underlay $\mathrm{CR}$ network with $M$ candidate relays.

\section{SySTEM MODEL}

\section{A. Problem Statement}

As shown in Fig. 1, on coexistence with PS in the downlink infrastructure mode, there are $M$ relays between the SU's transmitter (source) and receiver (destination). The SU's transmission pair as well as the relays can share the same frequency spectrum with the primary receiver; And a candidate relay is defined as the one which can forward the data packets for SU without violating the IT limit of $I_{t h}$. Also, there are two phases in the considered dual-hop cooperative network. During phase I, the SU's transmitter sends the data packet to the candidate relays, while it also produces a tolerable amount of interference to the PS's receiver at the same time. During phase II, one of these candidate relay can be selected to forward the received data packets to the destination. In this paper, we focus on how to select a relay during phase II so as to have a higher spectrum efficiency in terms of the TR value, which is defined as follows.

$$
T R=\frac{S S^{\prime} s \text { capacity gain }}{P S^{\prime} s \text { capacity loss }} .
$$

In the numerator, the SS's capacity gain means the capacity SS can achieve by sharing the spectrum resources with PS. Also, in the denominator, the PS's capacity loss stands for the capacity difference between the cases without and with the existence of SS. One should notice that a higher TR value means that the aforementioned PS's sacrifice is much worthier and a value lower than one is unacceptable in the underlay CR system.

In order to facilitate the performance analysis and clearly observe the impacts of low-interference relay selection on the system performance, several assumptions are made. First, following [13], the geographical proximity of these candidate relays are assumed such that they can experience the same channel statistics. Second, following the symmetry assumption in [14], equal distance is assumed for the link from the source to the group of relays, and that from the group of relays to the destination. Third, no direct link exists between the SU's transmitter and receiver. Fourth, in order to clarify the impacts of relay selection, constant transmission power of $P_{B}$ and $P_{S}$ are respectively assumed for the primary basestation (P-BS) and SS, including the SU's transmitter and group of relays [15]. At last, a certain channel estimation mechanism is necessary for evaluating the amount of intersystem interference from SS to PS (some discussions about the channel estimation can be found in Section III). However, designing an efficient channel estimation mechanism may beyond the scope of this paper.

\section{B. Signal Model}

Referring to the system model in Fig. 1, the received signals at the $i$-th relay, $y_{R_{i}}$, and destination, $y_{D}$, during Phases I and II can be written as

$$
y_{R_{i}}=\sqrt{P_{S}} \cdot h_{S R_{i}} \cdot x_{S}+\sqrt{P_{B}} \cdot h_{B R_{i}} \cdot x_{B 1}+n_{1}
$$

and

$$
y_{D}=\sqrt{P_{S}} \cdot h_{R_{i} D} \cdot x_{S}+\sqrt{P_{B}} \cdot h_{B D} \cdot x_{B 2}+n_{2},
$$

where $x_{S}$ with $\left|x_{S}\right|^{2}=1$ is the SU's transmitted signal; $x_{B 1}$ and $x_{B 2}$ with $\left|x_{B 1}\right|^{2}=\left|x_{B 2}\right|^{2}=1$ are the P-BS's transmitted signals during phases I and II, respectively. Following [13], the channel gains $h_{S R_{i}}, h_{R_{i} D}, h_{B R_{i}}$, and $h_{B D}$ are modeled as zero-mean complex Gaussian distributed random variables with variances $\sigma_{S R_{i}}^{2}, \sigma_{R_{i} D}^{2}, \sigma_{B R_{i}}^{2}$, and $\sigma_{B D}^{2}$, respectively. This also means that $\left|h_{S R_{i}}\right|^{2},\left|h_{R_{i} D}\right|^{2},\left|h_{B R_{i}}\right|^{2}$, and $\left|h_{B D}\right|^{2}$ are exponentially distributed random variables with means $\sigma_{S R_{i}}^{2}, \sigma_{R_{i} D}^{2}, \sigma_{B R_{i}}^{2}$, and $\sigma_{B D}^{2}$, respectively. The noise terms $n_{1}$ and $n_{2}$ are the zero-mean additive white Gaussian noise (AWGN) with variance $N_{0}$. Then, the SINR at the $i$-th relay and destination can be expressed as

$$
\gamma_{S R_{i}}=\frac{\left|h_{S R_{i}}\right|^{2} \cdot P_{S}}{\left|h_{B R_{i}}\right|^{2} \cdot P_{B}+N_{0}}
$$

and

$$
\gamma_{R_{i} D}=\frac{\left|h_{R_{i} D}\right|^{2} \cdot P_{S}}{\left|h_{B D}\right|^{2} \cdot P_{B}+N_{0}} .
$$

To simplify the expression and analysis in the later, we let $\eta_{S R_{i}} \triangleq\left|h_{S R_{i}}\right|^{2} P_{S} / N_{0}, \eta_{R_{i} D} \triangleq\left|h_{R_{i} D}\right|^{2} P_{S} / N_{0}, \eta_{B R_{i}} \triangleq$ $\left|h_{B R_{i}}\right|^{2} P_{B} / N_{0}$ and $\eta_{B D} \triangleq\left|h_{B D}\right|^{2} P_{B} / N_{0}$. Now, (4) and (5) can be rewritten as

$$
\gamma_{S R_{i}}=\frac{\eta_{S R_{i}}}{\eta_{B R_{i}}+1}
$$

and

$$
\gamma_{R_{i} D}=\frac{\eta_{R_{i} D}}{\eta_{B D}+1},
$$

where $\eta_{S R_{i}}, \eta_{R_{i} D}, \eta_{B R_{i}}$, and $\eta_{B D}$ are also exponentially distributed random variables with mean $\bar{\eta}_{S R_{i}} \triangleq \sigma_{S R_{i}}^{2} P_{S} / N_{0}$, $\bar{\eta}_{R_{i} D} \triangleq \sigma_{R_{i} D}^{2} P_{S} / N_{0}, \bar{\eta}_{B R_{i}} \triangleq \sigma_{B R_{i}}^{2} P_{B} / N_{0}$ and $\bar{\eta}_{B D} \triangleq$ $\sigma_{B D}^{2} P_{B} / N_{0}$, respectively.

\section{The Low-Interference Relay SElection Scheme}

Here, we propose a low-interference relay selection by taking the interference from relays to PS's receiver into account. The key idea is motivated by the pricing function in Game theory, which is usually defined as the difference between utility and cost functions to characterize the conflict of interests between users [12]. Thus, the low-interference selection rule can be defined as

$$
i_{L I}^{*}=\arg \max _{i=1 \sim M}\left(\gamma_{i}-\eta_{R_{i} P}\right),
$$

where the utility function $\gamma_{i}=\min \left(\gamma_{S R_{i}}, \gamma_{R_{i} D}\right)$ represents the bottleneck effect along the path of source, relay and destination; the cost function $\eta_{R_{i} P}=\left|h_{R_{i} P}\right|^{2} P_{S} / N_{0}$ denotes the normalized interference from the $i$-th relay toward the PS's receiver. Thus, a candidate relay is the one who can satisfy the IT limit i.e. $\eta_{R_{i} P}<I_{t h}$. Moreover, the order statistics of the independent and identically distributed (i.i.d) random variable 
$\gamma_{i} \forall i=1 \cdots M$ are the random variables $\gamma_{(i)} \forall i=1 \cdots M$, where $\gamma_{(1)}<\gamma_{(2)}<\cdots<\gamma_{(M)}$. Note that the conventional max-min selection can be conducted based on the rule of $i_{O P T}^{*}=\arg \max _{i=1 \sim M} \gamma_{i}$, which also gives $i_{O P T}^{*}=(M)$. In the following context, the optimal relay means the one selected by the conventional max-min policy.

One should notice that the cost of implementing the proposed relay selection scheme is the same as the conventional max-min scheme. In the underlay CR system, an SU should monitor the amount of interference he produced and keep it under the IT limit. That is to say each SU should have the ability of estimating the channel gain along the path from SU's transmitter to PU's receiver, i.e. $\left|h_{R_{i} P}\right|^{2}$, no matter which kind of relay selection is applied. However, designing an efficient channel estimation method may beyond the scope of this paper. In a word, the required information of the proposed scheme is the same as the conventional max-min scheme. Furthermore, the relay selection can be processed in a distributed way as demonstrated in [13], where each relay has a timer. Once a relay receives the Ready-to-Send (RTS) signal from the transmitter, it sets the timer inversely proportional to value of the selection criterion, e.g. $\gamma_{i}$ using the max-min scheme or $\gamma_{i}-\eta_{R_{i} P}$ using the proposed scheme. The relay who finishes the countdown procedure first can send the Clear-toSend (CTS) signal and forward the data packets.

\section{A. The Still Optimal Probability}

In order to investigate the impacts of the low-interference selection, the still optimal probability $P_{S O}$ can be defined as

$$
P_{S O}=\operatorname{Prob}\left[i_{L I}^{*}=i_{O P T}^{*}\right]=\operatorname{Prob}\left[i_{L I}^{*}=(M)\right],
$$

where $\operatorname{Prob}[E]$ evaluates the probability of event $E$. To facilitate the calculation of $P_{S O}$, we can also define

$$
\begin{aligned}
P_{i, j} & =\operatorname{Prob}\left[\left(\gamma_{(i)}-\eta_{R_{(i)} P}\right)>\left(\gamma_{(j)}-\eta_{R_{(j)} P}\right)\right] \\
& =\operatorname{Prob}\left[Q_{i, j}>A_{i, j}\right] \quad \forall(i)>(j),
\end{aligned}
$$

where $Q_{i, j}=\gamma_{(i)}-\gamma_{(j)}$ and $A_{i, j}=\eta_{R_{(i)} P}-\eta_{R_{(j)} P}$, respectively. Note that, as mentioned in the above, the ordering index $(i)$ is obtained by sorting $\gamma_{i} \forall i=1 \ldots M$ rather than $\gamma_{i}-\eta_{R_{i} P}$. Also, $Q_{i, j}>Q_{i, k}>0, \forall(j)+1 \leq(k) \leq(i)-1$ and $Q_{i, i}=0, \forall 1 \leq i \leq M$. Now, $P_{S O}$ can be rewritten as

$$
P_{S O}=P_{M, M-1} P_{M, M-2} \cdots P_{M, 2} P_{M, 1} .
$$

Moreover, for calculating the capacity in the later, we define $P_{\text {sel }}(i)$ as the probability of the selecting the $(i)$-th relay, i.e. the one with $\gamma_{(i)}$, by the low-interference selection as

$$
\begin{aligned}
P_{\text {sel }}(i)= & \left(1-P_{\text {sel }}(M)-P_{\text {sel }}(M-1)-\ldots-P_{\text {sel }}(i+1)\right) \times \\
& P_{i, i-1} P_{i, i-2} \ldots P_{i, 1} P_{i, 0},
\end{aligned}
$$

where $P_{1,0} \triangleq 1$. One should notice that $P_{\text {sel }}(M)=P_{S O}$ and $P_{\text {sel }}(1)+P_{\text {sel }}(2)+\cdots+P_{\text {sel }}(M-1)+P_{\text {sel }}(M)=1$. In the following, we derive $P_{i, j} \forall(i)>(j)$.

B. Statistics of $\gamma_{(i)}, Q_{i, j}$ and $A_{i, j}$

Recall the assumptions of geographical proximity and symmetry in Section II(A), we let $\bar{\eta}=\bar{\eta}_{S R_{i}}=\bar{\eta}_{R_{i} D}$ and $\bar{\eta}_{B R}=$ $\bar{\eta}_{B R_{i}} \forall i=1 \sim M$, respectively. After some derivations in [11], we can obtain the cumulative distribution function ( $c d f$ ) and probability density function $(p d f)$ of $\gamma_{i}$, i.e. $F_{\gamma_{i}}(r)$ and $f_{\gamma_{i}}(r)$, as follows

$$
\begin{aligned}
F_{\gamma}(r) & =F_{\gamma_{1}}(r)=\ldots=F_{\gamma_{M}}(r) \\
& =1-\frac{1}{\bar{\eta}_{B R} \bar{\eta}_{B D}}\left(\frac{r}{\bar{\eta}}+\frac{1}{\bar{\eta}_{B R}}\right)^{-1}\left(\frac{r}{\bar{\eta}}+\frac{1}{\bar{\eta}_{B D}}\right)^{-1} e^{-2 \frac{r}{\bar{\eta}}}
\end{aligned}
$$

and

$$
\begin{aligned}
f_{\gamma}(r)= & f_{\gamma_{1}}(r)=\ldots=f_{\gamma_{M}}(r) \\
= & {\left[\frac{1}{\bar{\eta}_{B R} \bar{\eta}_{B D}}\left(\frac{r}{\bar{\eta}}+\frac{1}{\bar{\eta}_{B R}}\right)^{-1}\left(\frac{r}{\bar{\eta}}+\frac{1}{\bar{\eta}_{B D}}\right)^{-1} e^{-2 \frac{r}{\bar{\eta}}}\right] } \\
& \times\left[\left(\frac{r}{\bar{\eta}}+\frac{1}{\bar{\eta}_{B R}}\right)^{-1}+\left(\frac{r}{\bar{\eta}}+\frac{1}{\bar{\eta}_{B D}}\right)^{-1}+2\right]
\end{aligned}
$$

Then, the $c d f$ and $p d f$ of the random variable $\gamma_{(i)} \forall i=1 \sim$ $M$ can now be expressed as

$$
\begin{aligned}
F_{\gamma_{(i)}}(r) & =P\left(\gamma_{(i)} \leq r\right) \\
& =\sum_{j=(i)}^{M} \frac{M !}{j !(M-j) !}\left(F_{\gamma}(r)\right)^{j}\left(1-F_{\gamma}(r)\right)^{M-j}
\end{aligned}
$$

and

$$
\begin{aligned}
f_{\gamma_{(i)}}(r)= & \frac{M !}{((i)-1) !(M-(i)) !}\left(F_{\gamma}(r)\right)^{(i)-1} \times \\
& \left(1-F_{\gamma}(r)\right)^{M-(i)} f_{\gamma}(r) .
\end{aligned}
$$

Furthermore, in [11], we derive the $p d f s$ of the random variables $Q_{i, j}$ and $A_{i, j}$ as

$$
\begin{aligned}
f_{Q_{i, j}}\left(q_{i, j}\right)= & G_{i j} \int_{0}^{\infty} F_{\gamma}^{(j)-1}(r) f_{\gamma}(r)\left[F_{\gamma}\left(r+q_{i, j}\right)-F_{\gamma}(r)\right]^{(i)-(j)-1} \\
& \times f_{\gamma}\left(r+q_{i, j}\right)\left[1-F_{\gamma}\left(r+q_{i, j}\right)\right]^{M-(i)} d r
\end{aligned}
$$

and

$f_{A_{i, j}}(a)=\frac{1}{2 \bar{\eta}_{R P}\left(1-e^{\left.-I_{t h} / \bar{\eta}_{R P}\right)^{2}}\right.}\left[e^{-\frac{|a|}{\bar{\eta}_{R P}}}-e^{\frac{|a|-2 I_{t h}}{\bar{\eta}_{R P}}}\right]$

where $q_{i, j} \geq 0,-I_{t h} \leq a \leq I_{t h}$ and $G_{i j}=$ $M ! /[((j)-1) !((i)-(j)-1) !(M-(\bar{i})) !], \quad$ respectively. Also, with $a \geq 0$, we can have the $c d f$ of $A_{i, j}$ as

$$
\begin{aligned}
F_{A_{i, j}}^{+}(a)= & 1-\frac{1}{\left(1-e^{\left.-I_{t h} / \bar{\eta}_{R P}\right)^{2}}\right.}\left[\frac{1}{2} e^{-\frac{a}{\bar{\eta}_{R P}}}+\right. \\
& \left.\frac{1}{2} e^{\frac{a-2 I_{t h}}{\bar{\eta}} R P}-e^{-\frac{I_{t h}}{\bar{\eta}_{R P}}}\right] \quad, \quad 0 \leq a \leq I_{t h} .
\end{aligned}
$$

Recall (10), we can now rewrite $P_{i, j}$ as

$$
P_{i, j}=\operatorname{Prob}\left[A_{i, j}<Q_{i, j}\right]=E_{Q_{i, j}}\left[F_{A_{i, j}}^{+}\left(q_{i, j}\right)\right]
$$

where $(i)>(j)$. To facilitate the derivation of $P_{i, j}$, we define

$$
f_{Q_{i, j}}\left(q_{i, j} \mid Q_{i, j}>q_{i, j+1}\right)=\frac{f_{Q_{i, j}}\left(q_{i, j}\right)}{\int_{q_{i, j+1}}^{\infty} f_{Q_{i, j}}(x) d x},
$$

where $q_{i, j}>q_{i, j+1}$ and $1 \leq(j) \leq(i)-1$. At last, the probability of $P_{i, j}$ can be calculated as

$$
\begin{aligned}
P_{i, j}= & \int_{0}^{\infty} \int_{q_{i, i-1}}^{\infty} \ldots \int_{q_{i, j+1}}^{\infty} F_{A_{i, j}}^{+}\left(q_{i, j}\right) f_{Q_{i, j}}\left(q_{i, j} \mid Q_{i, j}>q_{i, j+1}\right) \times \\
& f_{Q_{i, j+1}}\left(q_{i, j+1} \mid Q_{i, j+1}>q_{i, j+2}\right) \times \cdots \times \\
& f_{Q_{i, i-1}}\left(q_{i, i-1} \mid Q_{i, i-1}>q_{i, i}\right) d q_{i, j} d q_{i, j+1} \ldots d q_{i, i-1},(22)
\end{aligned}
$$

where $q_{i, i}=0$.

C. Capacity

The link capacity via the $(i)$-th relay can be defined as

$$
C(i)=\int_{0}^{\infty} \frac{1}{2} \log _{2}(1+x) f_{\gamma_{(i)}}(x) d x
$$

Then, the capacity of the low-interference and optimal relay selections, i.e. $C_{L I}$ and $C_{O P T}$, can be evaluated by $C_{O P T}=$ $C(M)$ and $C_{L I}=\sum_{i=1}^{M} P_{\text {sel }}(i) C(i)$, respectively. 


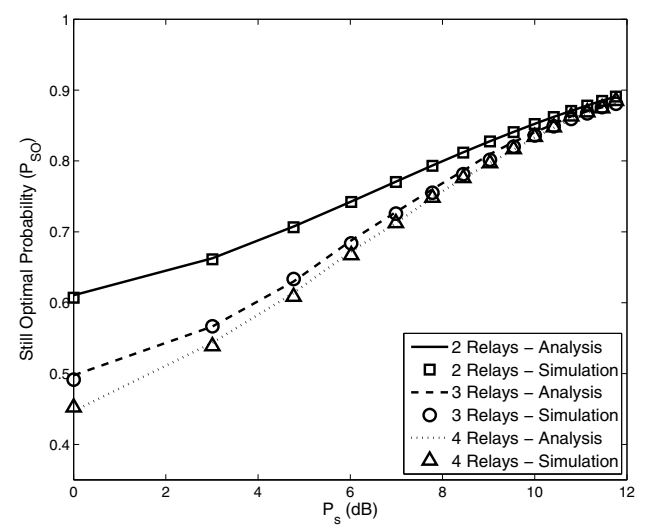

Fig. 2. The performance of $P_{S O}$ with respect to the transmission power $P_{S}$ for various number of candidate relays with $c_{g}=1$, where $I_{t h}=3 \mathrm{dBs}$ and $\bar{\eta}_{B R}=\bar{\eta}_{B D}=4 \mathrm{dBs}$.

\section{Numerical And Simulation Results}

In order to investigate the impacts and performance of the low-interference relay selection, we first present the performance of the still optimal probability $\left(P_{S O}\right)$, and then make some performance comparisons between the conventional max-min relay selection and proposed one in terms of capacity and TR value. In the simulations, the system topology is setup as shown in Fig. 1, where a single SU's transmission pair is considered to share the spectrum resource with one primary user (PU). Thus, in the following context, the PS's and SS's capacity are nominated as PU's and SU's capacity for more explicit illustration. The distances between P-BS and the group of relays $\left(d_{B R}\right), \mathrm{P}-\mathrm{BS}$ and the destination $\left(d_{B D}\right)$, P-BS and PU $\left(d_{B P}\right)$, and the groups of relay to PU $\left(d_{R P}\right)$ are set to be $d_{B R}=d_{B D}=2.5 d_{R D}, d_{B P}=1.5 d_{R D}$, and $d_{R P}=d_{R D}$, respectively, where $d_{R D}$ is the distance from the group of relays to the destination. To facilitate the following performance comparisons, we define $c_{g}=$ $E\left[\left|h_{R_{i} P}\right|^{2}\right] / E\left[\left|h_{R_{i} D}\right|^{2}\right]$. Following the assumptions in [13], $E\left[\left|h_{R_{i} D}\right|^{2}\right]=\sigma_{R D}^{2} \propto d_{R D}^{-\nu}$ and $E\left[\left|h_{R_{i} P}\right|^{2}\right]=\sigma_{R P}^{2} \propto d_{R P}^{-\nu}$ are assumed, respectively; $\nu$ is the path-loss exponent, which is assumed to be 3 . For simplicity, we let $d_{R D}=1$, which gives $c_{g}=\sigma_{R P}^{2} / \sigma_{R D}^{2}=\left(d_{R D} / d_{R P}\right)^{\nu}=\left(d_{R P}\right)^{-\nu}$. Also, unit variance is assumed for the AWGN, i.e. $N_{0}=1$.

\section{A. Still Optimal Probability $\left(P_{S O}\right)$}

Fig. 2 shows the performance of $P_{S O}$ with respect to the transmission power $P_{S}$ for various number of candidate relays with $c_{g}=1$, where $I_{t h}=3 \mathrm{dBs}$ and $\bar{\eta}_{B R}=\bar{\eta}_{B D}=4 \mathrm{dBs}$. From the figure, we can have several observations. First, the exactness of the analytical results are proved. Second, $P_{S O}$ decreases as the number of relay increases. With $P_{S}=3$ $\mathrm{dBs}, P_{S O}$ reduces from 0.66 to 0.54 when the number of relays increases from two to four. It is intuitional that when the number of candidate relay increases, the probability of picking a particular one reduces. Third, a larger $P_{S}$ can result in a smaller difference of $P_{S O}$ between the cases with variant number of relays. When we increase the transmission power, the first term of selecting a relay in (8). i.e. $\gamma_{i}$, becomes dominant. Note that the IT limit is fixed at $I_{t h}=3 \mathrm{dBs}$, which means $\eta_{R_{i} P}$ (the second term in (8)) should be less than $3 \mathrm{dBs}$. Therefore, in this situation, different number of relays can not lead to a significant difference of $\eta_{R_{i} P}$ compared to the larger value of $\gamma_{i}$. This reason also explains the positive relationship between the $P_{S O}$ and $P_{S}$.

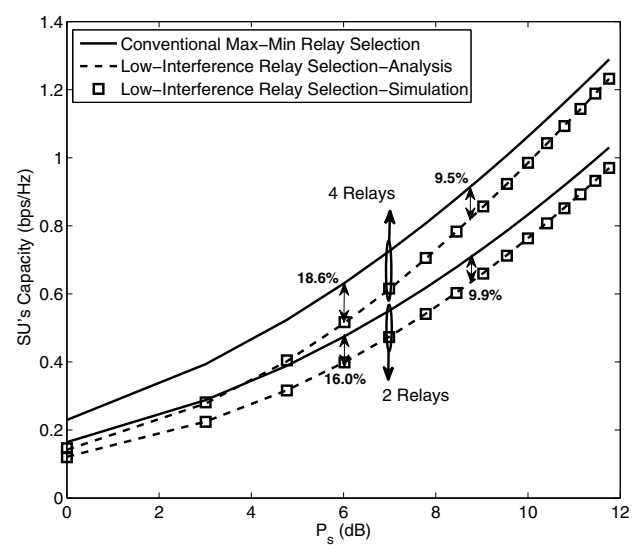

Fig. 3. The performance comparison between the proposed low-interference and conventional max-min relay selections with respect to the transmission power $P_{S}$ in terms of capacity, where $R_{t h}=0.25 \mathrm{bps} / \mathrm{Hz}, c_{g}=1, I_{t h}=3$ $\mathrm{dBs}$ and $\bar{\eta}_{B R}=\bar{\eta}_{B D}=4 \mathrm{dBs}$.

\section{B. Performance of Low-Interference Relay Selection}

Fig. 3 shows the performance comparison between the proposed low-interference and conventional max-min relay selections with respect to the transmission power $P_{S}$ in terms of capacity, where $R_{t h}=0.25 \mathrm{bps} / \mathrm{Hz}, c_{g}=1, I_{t h}=3$ $\mathrm{dBs}$ and $\bar{\eta}_{B R}=\bar{\eta}_{B D}=4 \mathrm{dBs}$. First, one can see that more relays can bring about some performance enhancements for not only the conventional max-min relay selection but also the proposed scheme. Observing Fig. 3 at $P_{S}=6 \mathrm{dBs}$, the capacity can raise from 0.40 to $0.52(\mathrm{bps} / \mathrm{Hz})$. The diversity gain obtained by using more relays accounts for this kind of performance improvement. Second, the performance gap between the conventional and proposed schemes become more obvious when there are more relays. For example, observing the SU's capacity with $P_{S}=6 \mathrm{dBs}$, the performance gaps are $18.6 \%$ and $16.0 \%$ for the cases of four and two relays, respectively. As shown in Fig. 2, the lower $P_{S O}$ associated with more relays can explain this phenomenon. Third, as $P_{S}$ increases, this performance gap and the difference of gap become less significant. One can see that the aforementioned performance gaps respectively reduce to $9.5 \%$ and $9.9 \%$ at $P_{S}=9 \mathrm{dBs}$, which results from the same reason as explained in the above for Fig. 2.

\section{Comparison of Capacity and Transfer Ratio (TR)}

Now, we compare capacity and TR values between the conventional max-min and proposed low-interference relay selections. In order to investigate the asymptotical behavior of the relay selections, the number of candidate relays is increased unpractically to 100 . Owing to the large number of relays, the numerical computation become time consuming. Thus, here, the comparison is made by simulation. In this simulation, the PU's capacity is fixed at four $\mathrm{bps} / \mathrm{Hz}$ for the case without the existence of SS. Figs. 4(a) and 4(b) show the performance comparison between the proposed lowinterference and conventional max-min relay selections with respect to various number of relays in terms of (a) capacity; and (b) transfer ratio (TR), where $c_{g}=1, I_{t h}=3 \mathrm{dBs}$, $\bar{\eta}=9 d B$ and $\bar{\eta}_{B R}=\bar{\eta}_{B D}=4 \mathrm{dBs}$. Apparently, one can find that although the SU's capacity can be degraded using the proposed scheme, the PU's capacity as well as the total capacity can be improved at the same cost as the conventional max-min selection (the cost issue is expounded in Section III). Observing Fig. 4(a) with five relays, the $7.5 \%$ loss of the SU's capacity can bring about $5 \%$ and $1.7 \%$ enhancement of PU's and total capacity. This is because the TR value is largely improved as shown in Fig. 4(b), which means that a 


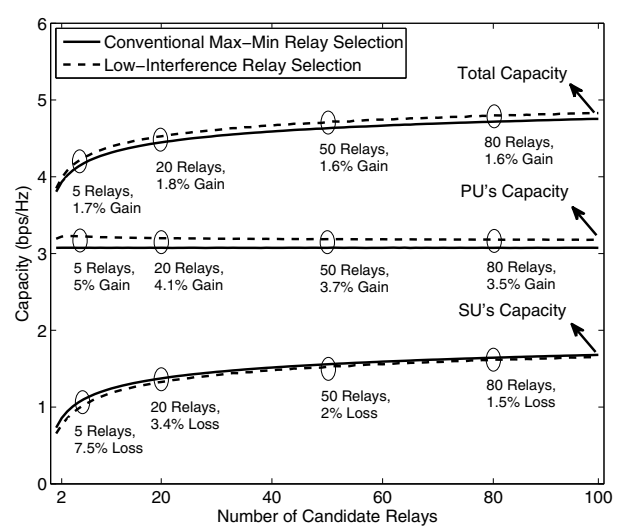

(a) Capacity

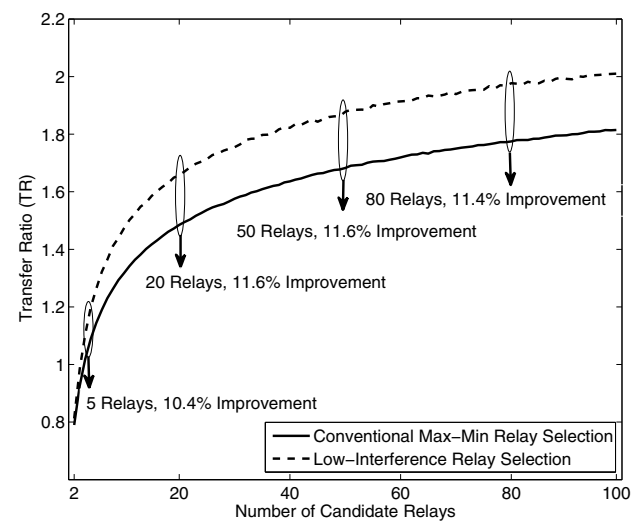

(b) Transfer Ratio (TR)

Fig. 4. The performance comparison between the proposed low-interference and conventional max-min relay selections with respect to various number of relays in terms of (a) capacity; and (b) transfer ratio (TR), where $c_{g}=1$, $I_{t h}=3 \mathrm{dBs}, \bar{\eta}=9 \mathrm{~dB}$ and $\bar{\eta}_{B R}=\bar{\eta}_{B D}=4 \mathrm{dBs}$. The PU's capacity is fixed at four $\mathrm{bps} / \mathrm{Hz}$ for the case without the existence of SS

higher spectrum efficiency can be achieved. Moreover, when the number of relays is increased to 100 , the capacity loss can be reduced to $1.5 \%$. Most importantly, the improvements in PU's capacity and total capacity can still be maintained, which proves the advantage of the proposed low interference relay selection.

\section{CONCLUSION AND Discussions}

In this paper, we have proposed a novel low-interference relay selection by considering the interference produced by the candidate relay toward the P-BS for the underlay $\mathrm{CR}$ networks using $\mathrm{DaF}$ cooperative transmission. To characterize the impacts of the low-interference selection, we coined the term still optimal probability $\left(P_{S O}\right)$ to indicate the chance of picking the optimal relay by the proposed selection strategy. Furthermore, we analytically investigated the performance of the low-interference relay selection in terms of the capacity. For the purpose of evaluating the overall spectrum efficiency, TR was defined as the ratio of the SS's capacity gain to the PS's capacity loss due to the activities of the SS. Via the analytical and simulation results, we can have the following observations. Firstly, raising the transmission power of the relay can also increase the chance of picking the optimal relay, i.e. a higher value of $P_{S O}$. Secondly, the performance of capacity can be improved as the number of relay increases. Thirdly, with a large number of candidate relays, the lowinterference selection can approximate the optimal one in terms of SU's capacity. Most importantly, a higher TR value as well as the total capacity can be obtained by the proposed scheme using the same channel information as the conventional max-min scheme, which indicates that a higher spectrum efficiency can be achieved without paying any extra costs.

This paper pioneers in evaluating the CR cooperative network using TR value by jointly taking the capacity of PS and SS into account. There are still more interesting and important topics for future. For example, the derivations and results of this paper depends on a given number of candidate relays. In reality, the number of candidate relays relies on several factors, e.g. the IT limit, available number of relays, environmental parameters, system topology, transmission power etc. These factors indeed can affect the TR value, too. Therefore, how to deploy a underlay CR system as well as the relay selection to own an optimal TR value can be an critical issue. Some self-organization algorithms to form a friendly relay set can also be an important topic. Last but the the least, a properly designed power control algorithm can increase the probability of a relay being an candidate one. Most importantly, jointly developing these techniques can achieve a higher TR value in other words, the higher spectrum efficiency. To be honest, the optimal TR relay selection is one of our on-going research topic, where we also prove that the optimal TR value is equivalent to the optimal capacity sum of PS and SS.

\section{REFERENCES}

[1] S. Haykin, "Cognitive radio: Brain-empowered wireless communications," IEEE Journal on Selected Areas in Communications, vol. 23, pp. 201-220, Feburary 2005.

[2] Q. Zhao and B. M. Sadler, "A survey of dynamic spectrum access," IEEE Signal Process. Mag., vol. 24, no. 3, pp. 79-89, May 2007.

[3] V. Chakravarthy, X. Li, Z. Wu, M. A. Temple, F. Garber, R. Kannan, and A. Vasilakos, "Novel overlay/underlay cognitive radio waveforms using sd-smse framework to enhance spectrum efficiency-part I: Theoretical framework and analysis in awgn channel," IEEE Trans. on Communiframework and analysis in awgn channel," IEEE Trans.
cations, vol. 57, no. 12, pp. 3794-3804, December 2009.

cations, vol. 57, no. 12, pp. 3794-3804, December 2009.
[4] R. Zhang, X. Kang, and Y. C. Liang, "Protecting primary users in cognitive radio networks: Peak or average interference power constraint?" in IEEE International Conference on Communications, Dresden, Germany, June 2009, pp. 1-5.

[5] S. Yan and X. Wang, "Power allocation for cognitive radio systems based on nonregenerative OFDM relay transmission," in International Confer-
ence on wireless communications, networking and mobile computing, Beijing, China, Sept. 2009, pp. 1-4

[6] C. Luo, F. R. Yu, H. Ji, and V. C. Leung, "Distributed relay selection and power control in cogitive radio networks with cooperative transmission," in IEEE International Conference on Communications, Cape Town, South Africa, May 2010, pp. 1-5.

[7] J. Mietzner, L. Lampe, and R. Schober, "Distributed transmit power allocation for multihop cognitive radio systems," IEEE Trans. on Wireless Communications, vol. 8, no. 10, pp. 5187-5201, Oct. 2009.

[8] M. Xie, W. Zhang, and K.-K. Wong, "A geometric approach to improve spectrum efficiency for cognitive relay networks," IEEE Trans. on Wireless Communications, vol. 9, no. 1, pp. 268-281, Jan. 2010.

[9] C. Sun and K. B. Letaief, "User cooperation in heterogeneous cognitive radio network with interference reduction," in IEEE International Conference on Communications, Beijing, China, May 2008, pp. 3193-3197.

[10] M. A. Beigi and S. M. Razavizadeh, "Cooperative beamforming in cognitive radio networks," in IFIP Wireless Days, Paris, France, Dec. 2009, pp. 1-5.

[11] C.W. (Wenson) Chang and P.-H. Lin, "A low-interference relay selection for decode-and-forward cooperative network in underlay cognitive radio," submitted to IEICE Trans. on Communications, April 2011.

[12] F. Meshkati, H. V. Poor, and S. C. Schwartz, "Energy-efficient resource allocation in wireless networks," IEEE signal processing magazine, vol. 24, no. 3, pp. 58-68, May 2010.

[13] A. Bletsas, A. Khisti, D. P. Reed, and A. Lippman, "A simple cooperative diversity method based on network path selection," IEEE Journal on Selected Areas in Communications, vol. 24, no. 3, pp. 659-672, May 2006.

[14] Y. Jing and H. Jafarkhani, "Single and multiple relay selection schemes and their achievable diversity orders" IEEE Transaction on Wireless Communications, vol. 8, no. 3, pp. 1414-1423, March 2009.

[15] L.-C. Wang and A. Chen, "Effects of location awareness on concurrent transmissions for cognitive ad hoc networks overlaying infrastructurebased systems," IEEE trans. mobile computing, vol. 8, no. 5, pp. 577589, May 2009. 